\title{
Propiedades psicométricas y resultados de la aplicación de la escala de disfrute y competencia percibida en baloncesto de iniciación*
}

\author{
Psychometric Properties and Results of Enjoyment and \\ Perceived Competence Scale in Youth Basketball
}

Recibido: mayo 28 de 2012 | Revisado: septiembre 9 de 2012 | Aceptado: diciembre 18 de 2012

\author{
JOSÉ LUIS ARIAS-ESTERO** \\ UCAM Universidad Católica San Antonio de Murcia, España

José IGNACIO ALONSO ****
JUAN LUIS YUSTE*****
Universidad de Murcia, España

doi:10.11144/Javeriana.UPSY12-3.ppra

Para citar este artículo: Arias-Estero, J. L., Alonso, J. I. \& Yuste, J. L. (2013). Propiedades psicométricas y resultados de la aplicación de la Escala de Disfrute y Competencia Percibida en baloncesto de iniciación. Universitas Psychologica, 12(3), 945-956. doi:10.11144/Javeriana.UPSY12-3.ppra

Artículo de investigación. Este trabajo es resultado del proyecto de investigación 15349/PHCS/10 financiado por la Fundación Séneca-Agencia de Ciencia y Tecnología de la Región de Murcia en el marco del II PCTRM 2007-2010.

** Universidad Católica San Antonio de Murcia, España. Correspondencia: José Luis Arias-Estero. Facultad de Ciencias de la Actividad Física y del Deporte. Universidad Católica San Antonio de Murcia. ResearcherID: C-1352-2009. Campus de Los Jerónimos, s/n. 30107 Guadalupe, Murcia, España.E-mail: jlae84@hotmail.com

**** Universidad de Murcia, España. Facultad de Educación. Campus de Espinardo, s/n. 30100 Espinardo, Murcia, España. E-mail: jialonso@um.es Researcher ID: Alonso, J. I. H-7919-2012

****** Universidad de Murcia, España. ResearcherID: H-6896-2013. Facultad de Educación. Campus de Espinardo, s/n. 30100 Espinardo, Murcia, España.E-mail: jlyuste@um.es

\section{RES UMEN}

Los objetivos de este estudio fueron: (a) conocer las propiedades psicométricas de una escala que permitiese medir el Disfrute y la Competencia Percibida en baloncesto de iniciación y (b) comprobar dichas medidas en esa misma población. Participaron 667 jóvenes $(M=10.62 ; D E=0.78)$, pertenecientes a 52 equipos. Doce expertos valoraron el contenido y 20 niños la comprensión. Se realizó un análisis factorial exploratorio, un análisis factorial confirmatorio y un análisis de fiabilidad mediante el alfa de Cronbach, el índice $h$ y el coeficiente de correlación intraclase. Los participantes mostraron valores muy altos, sin que existieran diferencias por razones de género o edad. El instrumento es válido y fiable para medir los dos factores en relación con la práctica de baloncesto en etapas de iniciación. Palabras clave autores

Motivación, iniciación deportiva, adherencia deportiva, enseñanza del deporte, validación.

Palabras clave descriptores

Psicometría, análisis factorial, validez, confiabilidad

\section{A B S T R A C T}

The aims of this study were: (a) to know the psychometric properties from a scale that allowed to measure enjoyment and perceived competence in youth basketball, and (b) to verify these measures in the same population. Sixty hundred sixty seven player $(M=10.62 ; S D=0.78)$ from 52 teams were participants. Twelve experts assessed the content and 20 children the understanding. An exploratory factor analysis, a confirmatory factor analysis and a reliability analysis using Cronbach's alpha, h index, and intraclass correlation coefficient were carried out. The tool is valid and reliable to measure youth basketball enjoyment and perceived competence. The participants showed higher values of enjoyment and perceived competence, without differences by gender or age.

Key words authors

Motivation, physical activity, youth sport, sport adherence, sport teaching, validation.

Key words plus

Psychometry, Factor Analysis, Validity, Reliability. 
Los resultados de diferentes estudios y encuestas señalan el aumento de niños sedentarios con problemas de obesidad y enfermedades cardiovasculares, que disminuyen su calidad de vida y aumentan los gastos sanitarios (p. ej., Ludwig, 2007; Ogden et al., 2006; Raitakari, Juonala \& Viikari, 2005). Los resultados del estudio enKid (Serra et al., 2003) estimaron que el $13.9 \%$ de la población española entre 2 y 24 años fue obesa y el $12.4 \%$ tenía sobrepeso. Ante esta realidad es necesario buscar soluciones para evitar el abandono prematuro de la práctica deportiva. Es acertado pensar que los factores motivacionales inciden en buena parte en los motivos de participación. Por lo que el afrontamiento de este problema desde un enfoque psicológico está plenamente justificado (Dishman et al., 2005; Moreno, Cervelló \& Martínez, 2007).

Estudios recientes corroboran que la participación de los niños en deportes organizados predice un aumento de la probabilidad de mantener estilos de vida físicamente activos durante la edad adulta (Anderssen, Wold \& Torsheim, 2005; Kjønniksen, Anderssen \& Wold, 2009; Telama, Yang, Hirvenssalo \& Raitakari, 2006). En este sentido, en baloncesto de iniciación, varios trabajos sugieren que la motivación de los niños por la práctica de este deporte podría mejorarse mediante la adaptación de las condiciones de juego a sus necesidades y posibilidades (Arias, Argudo \& Alonso, 2009; Chase, Ewing, Lirgg \& George, 1994). La adecuación de las condiciones de juego es una estrategia para crear hábitos de práctica deportiva durante la infancia y adolescencia. Esta medida posibilita que los niños puedan sentirse capaces de jugar y disfrutar de acuerdo con sus posibilidades. No obstante, no se han encontrado instrumentos adaptados a baloncesto de iniciación, que posibiliten obtener medidas del Disfrute y la Competencia Percibida de forma contextualizada.

La Teoría de la Autodeterminación (SDT) (Deci \& Ryan, 1985) considera que existen tres necesidades psicológicas básicas (autonomía, competencia y relación con los demás), que si manifiestan valores altos influyen positivamente en la motivación. En concreto, las personas que satisfacen estas necesidades muestran una mayor motivación intrínseca.
De lo contrario, lo probable es que se motiven de forma extrínseca (Moreno, Hernández \& González-Cutre, 2009). La mayor parte de los estudios indican que la motivación intrínseca está asociada con consecuencias más positivas que la extrínseca para mantenerse físicamente activo, como la vitalidad, autoestima, disfrute, interés, concentración, competencia, esfuerzo, persistencia y adherencia a la práctica (Cervelló, Escartí \& Balagué, 1999; MacDonald, Côté, Eys \& Deakin, 2011; Vallerand, 2007).

Dentro de los factores intrínsecos de la motivación en la SDT, el Disfrute y Competencia Percibida ocupan una posición importante en el desarrollo de la adherencia a la actividad deportiva (Brière, Vallerand, Blais \& Pelletier, 1995; Frederick \& Schuster-Schmidt, 2003). Una mayor percepción de Disfrute y Competencia se ha asociado a un incremento de la eficacia en la participación en programas deportivos regulares (Dishman et al., 2005), compromiso hacia la práctica (Álvarez, Balaguer, Castillo \& Duda, 2009) y mejora de las interacciones sociales intragrupo (Moreno et al., 2009). Los niños que disfrutan con lo que hacen y muestran mayor competencia percibida presentan una mayor predisposición a mantenerse practicando actividad física (Fraser-Thomas, Côté \& Deakin, 2008; Ullrich-French \& Smith, 2009; Wall \& Côté, 2007). El hecho de que un individuo, en las fases iniciales de una actividad deportiva, disfrute (Moreno, González-Cutre, Martínez, Alonso \& López, 2008; Scanlan \& Symons, 1992) y satisfaga las expectativas relacionadas con su competencia (Álvarez et al., 2009) debería asegurar la ausencia de abandonos.

Para poder evaluar estas dos dimensiones es necesario disponer de instrumentos adaptados al contexto donde se utilicen, con el fin de que los resultados sean válidos y fiables. Existen diversos instrumentos que han medido el Disfrute y la Competencia Percibida con la práctica deportiva, normalmente como dimensiones dentro de la motivación intrínseca. Duda y Nicholls (1992) establecieron una de las primeras herramientas básicas para la evaluación del disfrute (ocho ítems) y la competencia percibida (cuatro ítems) en situa- 
ciones deportivas con participantes de 15 años, aportando una escala que ha servido de guía para estudios sobre estas dimensiones. Cervelló (1996) adaptó al castellano ocho ítems de la Escala de Disfrute de Duda y Nicholls, para 134 deportistas adolescentes de ambos sexos, con un mínimo de 2 años de pertenencia a programas deportivos organizados. Otra de las escalas más utilizadas en el ámbito de la actividad física es la Physical Activity Enjoyment Scale ([PACES]; Kendzierirski \& DeCarlo, 1991). Se trata de una escala de disfrute muy potente psicométricamente, que se compone de 18 ítems de siete puntos. Esta fue modificada para una muestra de adolescentes por Molt et al. (2001), quedando compuesta por 16 ítems de cinco puntos. Posteriormente, Moreno et al. (2008) adaptaron con éxito la versión de Molt y colaboradores al contexto español para participantes de entre 12 y 54 años, con el fin de poder medir el disfrute deportivo. Una tercera escala habitualmente utilizada en este ámbito es la Intrisic Motivation Inventory ([IMI]; Plant \& Ryan, 1985). La versión original de la IMI se compone de 27 ítems de siete puntos que abarcan las dimensiones: (a) Disfrute, (b) Competencia Percibida, (c) Esfuerzo, (d) Presión y (e) Elección Percibida. Esta escala fue modificada por McAuley, Duncan y Tammen (1989) para su aplicación a una situación deportiva concreta: un juego popular de tiro en baloncesto. La escala modificada (consta de 18 ítems de siete puntos y solo abarca las cuatro primeras dimensiones de la original) reportó propiedades psicométricas adecuadas para 116 estudiantes universitarios con una edad media de 21.35 años.

Dada la relevancia del Disfrute y la Competencia Percibida en la investigación sobre motivación y adherencia a la práctica deportiva, los objetivos de este estudio fueron: (a) conocer las propiedades psicométricas (i. e., validez de contenido, comprensión y constructo, consistencia interna, fiabilidad y estabilidad temporal) de una escala preliminar que permitiese medir el Disfrute y la Competencia Percibida de forma adaptada a baloncesto de iniciación (BECS) y (b) y medir el disfrute y competencia percibida a través de la implementación de dicha escala en baloncesto de iniciación.

\section{Método}

\section{Participantes}

Participaron 667 (484 niños y 273 niñas) jóvenes jugadores de baloncesto de entre nueve y doce años $(M=10.62, D E=0.78)$, pertenecientes a 52 equipos participantes en categoría alevín, en las comunidades autónomas de Madrid, Murcia y Valencia. El muestreo fue intencionado, debido a la posibilidad de acceso a los participantes. La distribución de los participantes fue equilibrada en la variable edad $(50.2 \%$ de 9 a 10 años y $49.8 \%$ de 11 a 12 años) y no equilibrada en la variable género (65.9 \% niños y $34.1 \%$ niñas). El nivel de salud de los participantes fue normal y el nivel socioeconómico medio. Ellos habían jugado al baloncesto de forma federada durante $2.01(D E=0.95)$ años y a la semana practicaban una media de $5.21(\mathrm{DE}=1.05)$ horas, durante $3.21(D E=0.97)$ días. Los equipos estaban federados y jugaron a nivel autonómico. Todos los equipos participantes pertenecían a clubes y contaban con equipos en las categorías superiores.

\section{Procedimiento}

El trabajo de investigación se dividió en dos partes. Inicialmente, se adaptó la escala para baloncesto de iniciación y se evaluó la validez de contenido y comprensión. Posteriormente, se administró el instrumento a los participantes seleccionados para analizar la validez de constructo, consistencia interna, fiabilidad y estabilidad, y además para obtener información sobre el Disfrute y Competencia Percibida de los participantes.

\section{Adaptación inicial del instrumento}

El proceso de creación se dividió en cuatro etapas. En la primera, cuatro expertos ajenos al estudio (dos traductores profesionales y dos autores de reconocido prestigio internacional en el tema) realizaron una traducción de forma inversa (Hambleton, 1996) de los diez ítems correspondientes a las dimensiones Disfrute y Competencia Percibida 
de la escala IMI modificada por McAuley et al. (1989). De manera que dos expertos participaron en la primera traducción (del inglés al español) y otros dos en la segunda (del español al inglés). Se observó una total coincidencia al comparar ambas versiones en inglés.

En la segunda etapa, los autores del trabajo realizaron una adaptación, modificando diferentes términos con el fin de lograr una redacción apropiada a la población a la que se dirigía. También se modificó la escala original (Likert de 1 a 7) por otra cuyos valores de puntuación estaban comprendidos entre 1 (totalmente en desacuerdo) y 5 (totalmente de acuerdo).

En la tercera etapa, 12 jueces expertos valoraron la validez de contenido. Los expertos se seleccionaron intencionadamente con el fin de que cuatro fueran entrenadores de baloncesto en iniciación, con una experiencia previa superior a cinco años, y ocho profesores doctores afines al área de Educación Física y Deporte, pertenecientes a diferentes departamentos de diferentes universidades y con experiencia acreditada, como así denotaban sus currículums. De los doctores, cuatro eran especialistas en psicología, dos en sociología y dos en iniciación deportiva. A ellos se les solicitó que valorasen el grado de comprensión y adecuación de cada ítem y de la escala en general (cualitativa y cuantitativamente -escala del 0 al 10-), además de que realizasen sugerencias o propusiesen aspectos a mejorar. Siguiendo la propuesta de Bulger y Housner (2007), se eliminaron los ítems con valores inferiores a siete y se realizaron todas aquellas modificaciones sugeridas en el resto.

En la cuarta etapa, se realizó una reunión con 20 niños para obtener información acerca de la validez de comprensión de cada ítem. Este grupo fue seleccionado de manera intencionada, considerando que, a criterio de dos entrenadores, estaba formado por jugadores de bajo, medio y alto rendimiento. El investigador principal se reunió con este grupo de jugadores y les fue planteando las diez afirmaciones que conformaban la escala, de una en una, en una pizarra. Después de su lectura, ellos debían preguntar sobre las posibles dudas que les ocasionaba la afirmación para, a continuación, explicar al lado de cada ítem con sus palabras lo que significaba para ellos. Una vez escrita la explicación, cada uno la leía en voz alta. En el caso de que algún ítem no fuera comprendido, el investigador preguntaba a los jugadores sobre cómo solucionar la falta de comprensión.

\section{Administración del instrumento y obtención de datos}

Antes de administrar el cuestionario se obtuvo la autorización de los equipos, entrenadores y padres. La administración se realizó a cada equipo de forma independiente, durante los meses en que ellos competían los fines de semana. Cada equipo completó el cuestionario antes de un entrenamiento, bien en miércoles o jueves, sin la presencia del entrenador, pero sí de al menos de uno de los investigadores. Los ítems iban precedidos de la frase "Rodea el número sobre el grado de acuerdo con la frase, teniendo en cuenta que si rodeas el 1 "Estás totalmente en desacuerdo" con la frase, y si rodeas el 5 "Estás completamente de acuerdo" con la frase. Los jugadores no recibieron ninguna explicación adicional a la que aparecía en el propio instrumento. El tiempo requerido para completar el cuestionario fue inferior a cinco minutos. Una semana más tarde se volvió a repetir el procedimiento en circunstancias similares. Los cuestionarios de aquellos jugadores que faltaron a alguna de las dos tomas de datos fueron desechados.

\section{Análisis estadístico}

El análisis de los datos se realizó con los paquetes estadísticos PASWStatistics 19.0 y AMOS 7.0. La validez de contenido y comprensión se analizó mediante estadísticos descriptivos. La validez de constructo se estudió a través del análisis factorial exploratorio y confirmatorio; la consistencia interna, mediante el coeficiente $\alpha$ de Cronbach; la fiabilidad de la escala, utilizando el índice $h$ (Hancock \& Mueller, 2001; Li, Rosentahl \& Rubin, 1996) y la estabilidad, a través del coeficiente de correlación intraclase (ICC). El análisis factorial, el coeficiente $\alpha$ de Cronbach y el índice $h$ (utilizando la 
fórmula propuesta por Hancock \& Mueller, 2001), se calcularon con datos obtenidos de la primera medición. El análisis factorial confirmatorio se llevó a cabo empleando el método de estimación de máxima verosimilitud, y se obtuvo la normalidad multivariada mediante el índice de Mardia. Una vez realizada esta comprobación, se emplearon varios indicadores de ajuste del modelo $(\mathrm{Hu} \&$ Bentler, 1995). Concretamente, se utilizó la razón de chi cuadrado sobre los grados de libertad $\left(\chi^{2} / g l\right)$, el índice de ajuste comparativo (CFI), el índice de bondad de ajuste (GFI) y el error cuadrático medio de aproximación (RMSEA). Mediante la estadística descriptiva, también con los datos obtenidos en la primera medición, se aportó la medida de Disfrute y Competencia Percibida de los participantes, diferenciando los resultados según las variables independientes género y edad. Posteriormente, se utilizó el ANOVA factorial para analizar la relación entre las dos variables independientes (género y edad) y las dependientes (competencia percibida y disfrute). Con el fin de conocer la importancia del valor predictivo del disfrute y la competencia percibida a través del género y la edad se realizó un análisis de regresión logística. Por último, las pruebas efectuadas en relación con las medidas de disfrute y competencia percibida (ANOVA y regresión logística) fueron repetidas mediante el método bootstrapping para comprobar si los resultados eran similares a los encontrados con los datos originales. El nivel de significación estadística se estableció para $p<0.05$.

\section{Resultados}

\section{Propiedades psicométricas de la escala}

\section{Validación de contenido por expertos}

Los expertos valoraron la comprensión y adecuación de todos los ítems y globalmente de todo el cuestionario de manera bastante positiva (Tabla 1), aunque la estimación para los ítems 2, 7 y 9 estuvo por debajo de 7 . Los ítems 1,3 y 10 se mantuvieron en la escala sin sufrir ningún cambio, porque ninguno de los expertos comentó nada negativo al respecto. El ítem 7 fue eliminado porque, atendien- do a la sugerencia del experto 9, resultaba similar al primero ("Este ítem es igual al nํㅡ. No le veo sentido si no es como pregunta trampa"). Siete de los expertos manifestaron que las frases negativas de los ítems 2 y 9 generarían dudas sobre la respuesta. De manera que se optó por su eliminación. Con respecto al ítem 4, el experto 5 señaló que dudaba de que "Niños de las edades a las que está dirigido el instrumento comprendan lo que dice el ítem" y el experto 9 también resaltó "Me cuesta entender que se busca con "interesante"; si la ubico como dis-

\section{TABLA 1}

Medias y desviaciones estándar de la valoración de los expertos sobre la comprensión y adecuación del instrumento inicial

\begin{tabular}{|c|c|c|c|}
\hline Ítem & & Comprensión & Adecuación \\
\hline \multirow{2}{*}{1} & $M$ & 9.83 & 9.66 \\
\hline & DE & 1.94 & 2.05 \\
\hline \multirow{2}{*}{2} & $M$ & 6 & 6.91 \\
\hline & $D E$ & 3.01 & 2.54 \\
\hline \multirow{2}{*}{3} & $M$ & 9.41 & 9.58 \\
\hline & $D E$ & 1.44 & 0.79 \\
\hline \multirow{2}{*}{4} & M & 8.5 & 8.41 \\
\hline & $D E$ & 2.07 & 2.35 \\
\hline \multirow{2}{*}{5} & M & 7.75 & 7.75 \\
\hline & $D E$ & 1.95 & 2.56 \\
\hline \multirow{2}{*}{6} & $M$ & 8 & 8.5 \\
\hline & $D E$ & 1.47 & 1.56 \\
\hline \multirow{2}{*}{7} & $M$ & 6.41 & 6 \\
\hline & DE & 1.24 & 2.66 \\
\hline \multirow{2}{*}{8} & $M$ & 8.41 & 9 \\
\hline & $D E$ & 2.39 & 1.95 \\
\hline \multirow{2}{*}{9} & $M$ & 5.75 & 6.16 \\
\hline & $D E$ & 1.71 & 1.4 \\
\hline \multirow{2}{*}{10} & $M$ & 9.41 & 9.5 \\
\hline & $D E$ & 1.38 & 1.44 \\
\hline \multirow{2}{*}{ Valoración global } & M & 8.75 & 9 \\
\hline & $D E$ & 1.28 & 1.75 \\
\hline
\end{tabular}

Nota. 1: Disfruto mucho jugando al baloncesto, 2: No podría jugar muy bien al baloncesto, 3: Jugar al baloncesto es divertido, 4: Yo considero el baloncesto muy interesante, 5 : Después de jugar al baloncesto me siento bastante capaz, 6: Me siento bastante capaz cuando juego al baloncesto, 7: Mientras juego al baloncesto disfruto mucho, 8: Estoy satisfecho con mi rendimiento en baloncesto, 9: Cuando juego al baloncesto no consigo mantener la atención en el juego, 10: Creo que soy bastante bueno jugando al baloncesto.

Fuente: elaboración propia. 
frute (tanto como practicante como interesado en el juego, espectador)". Los expertos 1 y 7 realizaron sendas propuestas sobre la redacción de este ítem y finalmente se adoptó la del 6 en la versión final. Todos los expertos señalaron que los términos "capaz" y "rendimiento", que aparecían en los ítems 5, 6 y 8, no estaban adaptados a la capacidad de comprensión de los jugadores a estas edades. Cinco de los expertos hicieron propuestas para que los términos se sustituyeran por otros similares ("hábil", "bueno", "preparado", "nivel de juego"). Como ni los expertos ni los autores llegaron a un acuerdo sobre el término a utilizar, se adoptó la estrategia de no modificar los ítems hasta que el cuestionario pasara la validación de comprensión de los jugadores. Finalmente, como sugirió el experto 1 , se añadieron caras ilustrativas a los valores de la escala que sustituyeron al texto.

\section{Validación de comprensión por participantes}

El nivel de comprensión de los niños fue elevado. Solo los ítems 5, 6 y 8 les generaron dudas con respecto a los términos "capaz" y "rendimiento". Tras el diálogo que se estableció entre el investigador principal y el grupo de niños, se llegó a la conclusión de que era necesario equiparar los anteriores términos con "bueno" y "bien", respectivamente. De manera que estos ítems quedaron tal y como aparecen en la versión final de la BECS (ítems 1, 3 y 7; Figura 1).

\section{Validación de constructo}

Se comprobó que la medida de adecuación muestral de Kaiser-Meyer-Olkin $(\mathrm{KMO}=0.721)$ y la prueba de esfericidad de Bartlett $(p<0.001)$ aseguraban que los datos cumplían las condiciones necesarias para poder realizar el análisis factorial. El análisis factorial exploratorio de componentes principales produjo dos autovalores e indicó la extracción de dos factores. El factor Disfrute tuvo un autovalor de 4.26 y el análisis explicó el $39.94 \%$ de la varianza total generada por todos los ítems. El factor Competencia Percibida tuvo un autovalor de 4.52 y el análisis explicó el $46.06 \%$ de la varianza total generada por todos los ítems que componen la escala. Los valores hallados para cada uno de los

\begin{tabular}{ll}
\hline 00 & Totalmente en desacuerdo \\
\hline 00 & Algo en desacuerdo \\
\hline 00 & Ni de acuerdo ni en desacuerdo \\
\hline 00 & Algo de acuerdo \\
\hline 00 & Totalmente de acuerdo \\
\hline
\end{tabular}

\begin{tabular}{|c|c|c|c|c|c|}
\hline & $(0)$ & 0 & $\stackrel{0}{-}$ & $\underbrace{\bar{\theta}}$ & $\underbrace{\theta 0}$ \\
\hline 1. Después de jugar al baloncesto me siento bastante bueno & 1 & 2 & 3 & 4 & 5 \\
\hline 2. Disfruto mucho jugando al baloncesto & 1 & 2 & 3 & 4 & 5 \\
\hline 3. Me considero muy bueno cuando juego al baloncesto & 1 & 2 & 3 & 4 & 5 \\
\hline 4. Jugar al baloncesto es divertido & 1 & 2 & 3 & 4 & 5 \\
\hline 5. Estoy satisfecho con lo bien que juego al baloncesto & 1 & 2 & 3 & 4 & 5 \\
\hline 6. Considero el baloncesto un deporte muy interesante para practicar & 1 & 2 & 3 & 4 & 5 \\
\hline 7. Creo que soy bastante bueno jugando al baloncesto & 1 & 2 & 3 & 4 & 5 \\
\hline
\end{tabular}

Figura 1. Versión definitiva de la BECS.

Disfrute: 2, 4, 6. Competencia percibida: 1, 3, 5, 7 .

Fuente: elaboración propia. 
TABLA 2

Análisis factorial exploratorio de los dos factores de la Escala

\begin{tabular}{lc}
\hline \multicolumn{1}{c}{ Ítem } & Factor \\
\hline Disfrute & 0.832 \\
Disfruto mucho jugando al baloncesto. & 0.872 \\
Jugar al baloncesto es divertido. & 0.803 \\
Considero el baloncesto un deporte muy interesante para practicar. & \\
\hline Competencia percibida & 0.728 \\
Después de jugar al baloncesto me siento bastante bueno. & 0.87 \\
Me considero muy bueno cuando juego al baloncesto. & 0.79 \\
Estoy satisfecho con lo bien que juego al baloncesto. & 0.878 \\
Creo que soy bastante bueno jugando al baloncesto. & \\
\hline
\end{tabular}

Fuente: elaboración propia.

ítems presentaron saturaciones entre 0.8 y 0.87 para el factor Disfrute y entre 0.73 y 0.87 para el factor Competencia Percibida (Tabla 2).

Los resultados del análisis de la normalidad multivariada (coeficiente de Mardia $=59.3$ ) permitieron aceptar que los datos obtenidos de las estimaciones no se vieron afectados por la falta de normalidad (Byrne, 2010). Los indicadores mostraron una buena bondad de ajuste para el modelo disfrute $\left(\chi^{2} / g l=4.9 ; \mathrm{CFI}=0.92 ; \mathrm{GFI}=0.9 ; \mathrm{RMSEA}\right.$ $=0.06)$ y para el modelo competencia percibida $\left(\chi^{2}\right)$ $g l=4 ; \mathrm{CFI}=0.95 ; \mathrm{GFI}=0.94 ; \mathrm{RMSEA}=0.04)$.

\section{Consistencia interna, fiabilidad}

y estabilidad temporal

Se obtuvieron coeficientes de fiabilidad alfa de Cronbach de 0.88 para Disfrute y 0.88 para Competencia Percibida, revelando una buena consistencia interna. Además, dado que los ítems no mostraron valores de la correlación elemento-total corregida por debajo de 0.4 (Tabla 3), no fue necesario eliminar ninguno de ellos (Ebel \& Frisbie, 1986). El índice $h$ (Hancock \& Mueller, 2001; Li et al., 1996) proporcionó un valor de 0.9 , indicando así una fia-

TABLA 3

Análisis del índice de discriminación de la Escala

\begin{tabular}{|c|c|c|c|c|}
\hline Ítem & $\begin{array}{l}\text { Media si se elimina } \\
\text { el elemento }\end{array}$ & $\begin{array}{l}\text { Varianza si se } \\
\text { elimina el elemento }\end{array}$ & $\begin{array}{l}\text { Correlación elemento- } \\
\text { total corregida }\end{array}$ & $\begin{array}{l}\text { alfa de Cronbach si se } \\
\text { elimina el elemento }\end{array}$ \\
\hline $\begin{array}{l}\text { Disfruto mucho jugando al } \\
\text { baloncesto. }\end{array}$ & 26.66 & 7.653 & 0.479 & 0.696 \\
\hline $\begin{array}{l}\text { Jugar al baloncesto es } \\
\text { divertido. }\end{array}$ & 26.72 & 7.604 & 0.443 & 0.702 \\
\hline $\begin{array}{l}\text { Considero el baloncesto un } \\
\text { deporte muy interesante para } \\
\text { practicar. }\end{array}$ & 26.72 & 7.533 & 0.492 & 0.694 \\
\hline $\begin{array}{l}\text { Después de jugar al } \\
\text { baloncesto me siento } \\
\text { bastante bueno. }\end{array}$ & 27.07 & 6.415 & 0.576 & 0.65 \\
\hline $\begin{array}{l}\text { Me considero muy bueno } \\
\text { cuando juego al baloncesto. }\end{array}$ & 27.3 & 5.454 & 0.661 & 0.621 \\
\hline $\begin{array}{l}\text { Estoy satisfecho con lo bien } \\
\text { que juego al baloncesto. }\end{array}$ & 27.11 & 5.798 & 0.559 & 0.656 \\
\hline $\begin{array}{l}\text { Creo que soy bastante bueno } \\
\text { jugando al baloncesto. }\end{array}$ & 27.19 & 5.564 & 0.658 & 0.622 \\
\hline
\end{tabular}

Fuente: elaboración propia. 
bilidad elevada. La fiabilidad temporal de la escala también fue alta $(\mathrm{ICC}=0.85)$.

\section{Medidas de Disfrute y Competencia percibida}

Los participantes del presente estudio declararon un Disfrute $(M=4.44 ; D E=0.41)$ y una Competencia percibida $(M=4.49 ; D E=0.41)$ de tendencia elevada, considerando un rango de valores mínimos y máximos de 1 a 5 , respectivamente (Tabla 4). Los resultados del nivel crítico asociado al estadístico $F$ de la prueba ANOVA factorial, indicaron que el modelo no explicó una parte significativa de la variación observada en la Competencia percibida $\left(F=0.498 ; p=0.684 ; \eta^{2}=0.002\right)$ ni en el Disfrute $\left(F=0.576 ; p=0.321 ; \eta^{2}=0.007\right)$.

Con respecto a la variable género, no se hallaron diferencias estadísticamente significativas entre los niños y las niñas en cuanto a Disfrute y Competencia Percibida (Disfrute: $F=0.036 ; p=0.849$; $\eta^{2}=0.000$ y Competencia Percibida: $F=0.098$ $\left.p=0.754 ; \eta^{2}=0.000\right)$. Respecto a la variable edad, tampoco se encontraron diferencias estadísticamente significativas entre los participantes agrupados en el rango de 11 a 12 años en comparación con los agrupados en el rango de 9 a 10 años (Disfrute: $F=0.126 ; p=0.149 ; \eta^{2}=0.000$ y Competencia Percibida: $F=1.088 ; p=0.297 ; \eta^{2}=0.000$ ).

El análisis de regresión logística reportó que ni el género ni la edad afectaron al Disfrute (género:
$B=-0.068 ; E T=0.197 ; p=0.728$ y edad: $B=$ $-0.103 ; E T=0.188 ; p=0.581)$ ni a la Competencia percibida (género: $B=-0.076 ; E T=0.177 ; p=$ 0.635 y edad: $B=-0.099 ; E T=0.165 ; p=0.421$ ). Estos resultados confirmaron los obtenidos mediante el análisis de la varianza factorial.

Los valores de significación estadística, obtenidos mediante el método bootstrapping en todos los casos analizados en relación con las medidas de Disfrute y Competencia percibida, fueron similares a los derivados del ANOVA factorial y de la regresión logística con la muestra original.

\section{Discusión}

Los objetivos de este estudio fueron: conocer las características psicométricas de la escala BECS para su aplicación con jugadores en la etapa de iniciación al baloncesto y medir el Disfrute y la Competencia Percibida en esa misma población. En cuanto al primer objetivo, el instrumento se mostró válido y fiable para medir el Disfrute y la Competencia Percibida en la iniciación al baloncesto. Además, contó con la garantía de la validez de contenido de los expertos y de comprensión de los participantes. En cuanto al segundo objetivo, el total de los participantes declararon un Disfrute y una Competencia Percibida de tendencia alta.

El análisis factorial exploratorio reveló que todos los ítems se agruparon en dos factores. El valor ha-

TABLA 4

Estadísticos descriptivos de Disfrute y Competencia percibida

\begin{tabular}{cccccc}
\hline \multirow{2}{*}{ Género } & \multirow{2}{*}{ Edad } & \multicolumn{2}{c}{ Disfrute } & \multicolumn{2}{c}{ Competencia percibida } \\
\cline { 3 - 6 } Niño & 9 a 10 años & 4.78 & 4.52 & 4.52 & DE \\
& 11 a 12 años & 4.66 & 4.48 & 4.48 & 0.41 \\
& Total & 4.45 & 4.5 & 4.5 & 0.45 \\
\hline \multirow{2}{*}{ Niña } & 9 a 10 años & 4.67 & 4.47 & 4.47 & 0.42 \\
& 11 a 12 años & 4.7 & 4.5 & 4.5 & 0.34 \\
& Total & 4.68 & 4.49 & 4.49 & 0.38 \\
\hline \multirow{2}{*}{ Total } & 9 a 10 años & 4.76 & 4.5 & 4.5 & 0.41 \\
& 11 a 12 años & 4.66 & 4.48 & 4.48 & 0.41 \\
& Total & 4.44 & 4.49 & 4.49 & 0.41 \\
\hline
\end{tabular}

Fuente: elaboración propia. 
llado en cada uno de los ítems presentó saturaciones superiores a 0.8 para el Disfrute y a 0.7 para la Competencia Percibida, lo cual según Hair, Anderson, Tatham y Black (1998) significa que identificaron muy bien el factor y fueron de una clara relevancia práctica. Resultados similares fueron encontrados en el estudio de Duda y Nicholls (1992) para Disfrute, aunque estos resultados fueron superiores a los de Moreno et al. (2008) con respecto al Disfrute y a los de Duda y Nicholls para la Competencia Percibida. Además, la estructura de ambos factores explicó un alto porcentaje de la varianza del instrumento (Hair et al., 1998). El valor obtenido para el factor Disfrute fue inferior a los obtenidos en el trabajo de Duda y Nicholls con 119 practicantes de actividades deportivas (49.5\%), pero similar al $40.42 \%$ reportado por Moreno y colaboradores. El valor obtenido para el factor Competencia percibida fue superior al $7.4 \%$ obtenido por Duda y Nicholls.

El análisis factorial confirmatorio resultó aceptable para ambos modelos, debido a que el valor de $\chi^{2} / g l$ fue inferior a cinco, los índices CFI y GFI se aproximaron al valor óptimo de uno y el índice RMSEA fue inferior o igual a $0.05(\mathrm{Hu} \&$ Bentler, 1995). El análisis factorial confirmatorio también fue adecuado en los estudios previos de validación de la escala IMI adaptada al baloncesto (McAuley et al., 1989) y en las escalas cuyo único factor analizado fue el Disfrute (Molt et al., 2001; Moreno et al., 2008).

El valor de alfa de Cronbach en ambos factores denotó una alta consistencia interna, según considera la literatura especializada (Carretero-Dios \& Pérez, 2005; Hair et al., 1998). El resultado para el factor Disfrute fue superior al 0.8 alcanzado por McAuley et al. (1989) con la escala IMI adaptada al baloncesto y similar al 0.89 de la escala PACES (Moreno et al., 2008). El resultado para el factor Competencia Percibida también fue similar al 0.87 obtenido por McAuley et al. (1989). La fiabilidad y la estabilidad temporal también fueron buenas, lo cual aportó mayor fuerza a los resultados globales del instrumento con respecto a la fiabilidad.

En relación con las medidas de Disfrute y Competencia Percibida, los resultados fueron superiores a los obtenidos por McAuley et al. (1989) tras uti- lizar la escala (Likert de 1 a 7) con 116 estudiantes universitarios con una edad media de 21.35 años $(\mathrm{M}$ Disfrute $=4.77 ; \mathrm{M}$ Competencia Percibida $=$ 4.37). La diferencia pudo deberse a que los participantes del estudio de McAuley et al. (1989) fueron universitarios. Según la literatura consultada (García Ferrando, 2006; Pavón, 2004), en el ámbito universitario, el interés por participar en actividades físicas está influido por razones relacionadas con el mantenimiento de la forma física y la mejora de la salud, mientras que en edades más tempranas predomina el disfrute con la práctica deportiva.

El Disfrute y la Competencia Percibida son factores que sobresalen de entre los más relevantes, para determinar la continuidad de la práctica de actividad física en la edad adulta (Brustad, 1993; Csikszentmihalyi, 1996). Atendiendo a la literatura consultada (Anderssen et al., 2005; Kjønniksen et al., 2009; Telama et al., 2006) y a partir de los altos valores de Disfrute y Competencia Percibida, se podría inferir que los participantes de este estudio tendrían altas posibilidades de mantener estilos de vida físicamente activos durante la edad adulta. No obstante, aunque el disfrute y la competencia percibida sean factores ligados a la motivación intrínseca por la práctica deportiva, sería deseable que en el futuro se evaluase si el efecto se produciría en la práctica real.

En el presente trabajo, las variables edad y género no influyeron en la predicción de Disfrute y Competencia Percibida. Este resultado sigue la línea defendida por García Ferrando (2006), en cuanto a que las diferencias que se establecen entre los sujetos por razones de género aparecen progresivamente a partir de la adolescencia, como consecuencia de la influencia de los agentes sociales que rodean a los niños (p. ej., familia, escuela, amigos). Esta misma tendencia, con respecto a la edad, fue señalada por Gray, Sproule y Wang (2008) con alumnos de primaria y secundaria. Sin embargo, los resultados con respecto al género no fueron similares debido a que, a diferencia de lo que ocurrió en el presente trabajo, en el estudio de Gray y colaboradores los niños reportaron mayores valores de Disfrute y Competencia Percibida que las niñas. La no 
diferenciación por género en la presente investigación podría deberse a que los participantes fueron jugadores de baloncesto, que en principio lo practicaban de manera voluntaria, a diferencia del estudio de Gray et al. (2008). Según Chen y Darst (2002) el nivel de desarrollo de las habilidades y el interés por un deporte influye en el Disfrute y la Competencia Percibida de los participantes. A partir de la anterior afirmación, al ser los participantes jugadores de baloncesto, cabría esperar que tuvieran unos puntajes similares de Disfrute y Competencia percibida.

En conclusión, este estudio presenta un instrumento válido y fiable para conocer, concretamente, el disfrute y la competencia percibida en baloncesto en etapas de iniciación. Las propiedades adecuadas de esta sencilla escala posibilitan su uso tanto en nuevas investigaciones como en el contexto deportivo. No obstante, la principal limitación en relación con la obtención de las propiedades psicométricas fue que no se relacionaron los constructos de la escala propuesta con otros de la teoría de la SDT (e. g., motivación autodeterminada) para aportar mayor evidencia de validez concurrente y de constructo. Por lo que son necesarias nuevas investigaciones que reafirmen la validez y fiabilidad del instrumento aquí presentado. Por otro lado, los participantes del presente estudio mostraron unos valores de disfrute y competencia percibida muy altos, sin que existieran diferencias por razones de género o edad. En función de esta conclusión, serían necesarios nuevos estudios que analizaran variables que pudiesen influir en que los jugadores de baloncesto, en etapas de iniciación, mantuviesen valores altos de competencia percibida y disfrute. Numerosos factores y variables de naturaleza psicológica pueden mediar en esta relación. La adaptación de las condiciones de juego a las posibilidades de los jugadores podría ser una estrategia a tener en cuenta. En este sentido, Villamarín, Maurí y Sanz (1998) demostraron que se puede intervenir sobre una mejora de la estructuración de los entrenamientos y del diseño de situaciones motrices teniendo en cuenta los factores competencia percibida y disfrute.

\section{Referencias}

Álvarez, M. S., Balaguer, I., Castillo, I. \& Duda, J. (2009). Coach autonomy support and quality of sport engagement in young soccer players. The Spanish Journal of Psychology, 12(1), 138-148.

Anderssen, N., Wold, B. \& Torsheim, T. (2005). Tracking of exercise in adolescence. Research Quarterly for Exercise ES Sport, 76(2), 119-129.

Arias, J. L., Argudo, F. M. \& Alonso, J. I. (2009). Effect of the three-point line change on the game dynamics in girls' mini-basketball. Research Quarterly for Exercise and Sport, 80(3), 502-509.

Brière, N., Vallerand, R., Blais, M. \& Pelletier, L. (1995). Development and validation of a measure of intrinsic, extrinsic and amotivation in sports: The Sport Motivation Scale (SMS). International Journal of Sport Psychology, 26(4), 465-489.

Brustad, R. J. (1993). Who will go out and play? Parental and psychological influences on children's attraction to physical activity. Pediatric Exercise Science, 5(3), 210-223.

Bulger, S. M. \& Housner, L. D. (2007). Modified delphi investigation of exercise science in physical education teacher education. Journal of Teaching in Physical Education, 26(1), 57-80.

Byrne, B. M. (2010). Structural equation modeling with Amos: Basic concepts, applications, and programming ( $2^{\mathrm{a}}$ ed.). New York: Routledge.

Carretero-Dios, H. \& Pérez, C. (2005). Normas para el desarrollo y revisión de estudios instrumentales. International Journal of Clinical and Health Psycho$\log y, 5(3), 521-551$.

Cervelló, E. (1996). La motivación y el abandono deportivo desde la perspectiva de las metas de logro. Tesis Doctoral, Universidad de Valencia, Valencia, España.

Cervelló, E., Escartí, A. \& Balagué, G. (1999). Relaciones entre la orientación de meta disposicional y la satisfacción con los resultados deportivos, las creencias sobre las causas de éxito en el deporte y la diversión con la práctica deportiva. Revista de Psicología del Deporte, 8(1), 7-19.

Chase, M. A., Ewing, M. E., Lirgg, C. D. \& George, T. R. (1994). The effects of equipment modification on children's self-efficacy and basketball shooting 
performance. Research Quarterly for Exercise $\mathcal{B}^{2}$ Sport, 65(2), 159-168.

Chen, A. \& Darst, P. W. (2002). Individual and situational interest: The role of gender and skill. Contemporary Educational Psychology, 27(2), 250-269. doi: 10.1006/ceps.2001.1093

Csikszentmihalyi, M. (1996). Creativity. New York: Harper Collins.

Deci, E. L. \& Ryan, R. M. (1985). The general causality orientations scale: Self-determination in personality. Journal of Research in Personality, 19(2), 109-134.

Dishman, R., Molt, R., Saunders, R., Felton, G., Ward, D., Dowda, M., et al. (2005). Enjoyment mediates effects of a school-based physical-activity intervention. Medicine $\mathcal{E}$ Science in Sports $\mathcal{E}$ Exercise, 37(3), 478-487.

Duda, J. L. \& Nicholls, J. G. (1992). Dimensions of achievement motivation in schoolwork and sport. Journal of Educational Psychology, 84(3), 290-299.

Ebel, R. L. \& Frisbie, D. A. (1986). Essentials of education measurement. Englewood Cliffs, NJ: Prentice Hall.

Fraser-Thomas, J., Côté, J. \& Deakin, J. (2008). Examining adolescent sport dropout and prolonged engagement from a developmental perspective. Journal of Applied Sport Psychology, 20(3), 318-333. doi: 10.1080/10413200802163549

Frederick, C. M. \& Schuster-Schmidt, H. (2003). Competition and intrinsic motivation in physical activity: A comparison of two groups. Journal of Sport Behavior, 26(3), 240-254.

García Ferrando, M. (2006). Posmodernidad y deporte: entre la individualización y la masificación. Encuesta hábitos deportivos de los españoles 2005. Madrid: CSD/CIS.

Gray, S., Sproule, J. \& Morgan, K. (2008). Teaching team invasion games and motivational climate. European Physical Education Review, 15(1), 65-89. doi: 10.1177/1356336X09105212

Hair, J. F., Anderson, R. E., Tatham, R. L. \& Black, W. C. (1998). Multivariate data analysis ( $5^{\mathrm{a}} \mathrm{ed}$.). Upper Saddle River, NJ: Prentice Hall.

Hambleton, R. K. (1996). Adaptación de tests para su uso en diferentes idiomas y culturas: fuentes de error, posibles soluciones y directrices prácticas. En J. Muñiz (Ed.), Psicometría (pp. 207-238). Madrid: Universitas.
Hancock, G. R. \& Mueller, R. O. (2001). Rethinking construct reliability within latent variable systems. En R. Cudeck, S. du Toit \& D. Soerbom (Eds.), Structural equation modeling: Present and future. A festschrift in honor of Karl Jöreskog (pp. 195-216). Lincolnwood, IL: Scientific Software International.

Hu, L. \& Bentler, P. (1995). Evaluating model fit. En R. Hoyle (Ed.), Structural equation modelling: Concepts, issues and applications (pp. 76-99). Thousand Oaks, CA: Sage.

Kendzierirski, D. \& DeCarlo, K. L. (1991). Physical activity enjoyment scale: Two validation studies. Journal Sport and Exercise Psychology, 13(1), 50-64.

Kjønniksen, L., Anderssen, N. \& Wold, B. (2009). Organized youth sport as a predictor of physical activity in adulthood. Scandinavian Journal of Medicine $\mathcal{E}$ Science in Sports, 19(5), 646-654. doi: 10.1111/j.1600-0838.2008.00850.x

Li, H., Rosenthal, R. \& Rubin, D. B. (1996). Reliability of measurement in psychology: From Spearman-Brown to maximal reliability. Psychological Methods, 1(1), 98-107.

Ludwig, D. S. (2007). Childhood obesity: The shape of things to come. New England Journal of Medicine, 357(23), 2325-2327.

MacDonald, D., Côté, J., Eys, M. A. \& Deakin, J. (2011). The role of enjoyment and motivational climate in relation to the personal development of team sport athletes. The Sport Psychologist, 25(1), 32-46.

McAuley, E., Duncan, T. \& Tammen, V. V. (1989). Psychometric properties of the intrinsic motivation inventory in a competitive sport setting: A confirmatory factor analysis. Research Quarterly for Exercise $\mathcal{E}$ Sport, 60(1), 48-58.

Molt, R. W., Dishman, R., Saunders, R., Dowda, M., Felton, G. \& Pate, R. R. (2001). Measuring enjoyment of physical activity among adolescent girls. American Journal of Preventive Medicine, 21(2), 110-117.

Moreno, J. A., Cervelló, E. \& Martínez, A. (2007). Measuring self-determination motivation in a physical fitness setting. Validation of the Behavioural Regulation in Exercise Questionnaire-2 (BREQ-2) in a Spanish sample. Journal of Sports Medicine and Physical Fitness, 47(3), 366-378. 
Moreno, J. A., González-Cutre, D., Martínez, C., Alonso, N. \& López, M. (2008). Propiedades psicométricas de la Physical Activity Enjoyment Scale (PACES) en el contexto español. Estudios de Psicología, 29(2), 173-180.

Moreno, J. A., Hernández, A. \& González-Cutre, D. (2009). Complementando la teoría de la autodeterminación con las metas sociales: un estudio sobre la diversión en educación física. Revista Mexicana de Psicología, 26(2), 213-222.

Ogden, C. L., Carroll, M. D., Curtin, L. R., McDowell, M. A., Tabak, C. J. \& Flegal, K. M. (2006). Prevalence of overweight and obesity in the United States, 1999-2004. Journal of the American Medical Association, 295(13), 1549-1555. doi: 10.1001/ jama.295.13.1549

Pavón, A. (2004). Motivaciones e intereses de los universitarios murcianos hacia la práctica físico-deportiva. Tesis Doctoral, Universidad de Murcia, España.

Plant, R. \& Ryan, R. M. (1985). Self-consciousness, self-awareness, ego-involvement, and intrinsic motivation: An investigation of internally controlling styles. Journal of Personality, 53(3), 435-449. doi: 10.1111/j.1467-6494.1985.tb00375.x

Raitakari, O. T., Juonala, M. \& Viikari, J. S. (2005). Obesity in childhood and vascular changes in adulthood: Insights into the Cardiovascular Risk in Young Finns Study. International Journal of Obesity, 29(1), 101-104. doi: 10.1038/sj.ijo.0803085
Scanlan, T. K. \& Symons, J. P. (1992). The construct of enjoyment. En G. C. Roberts (Ed.), Motivation in sport and exercise (pp. 199-215). Champaign, IL: Human Kinetics.

Serra, L., Ribas, L., Aranceta, J., Pérez, C., Saavedra, P. \& Peña, L. (2003). Obesidad infantil y juvenil en España. Resultados del Estudio enKid (19982000). Medicina Clínica, 121(19), 725-732.

Telama, R., Yang, X., Hirvenssalo, M. \& Raitakari, O. (2006). Participation in organized youth sport as a predictor of adult physical activity: A 21-year longitudinal study. Pediatric Exercise Science, 18(1), 76-88.

Ullrich-French, S. \& Smith, A. L. (2009). Social and motivational predictors of continued youth sport participation. Psychology of Sport and Exercise, 10(1), 87-95.

Vallerand, R. J. (2007). Intrinsic and extrinsic motivation in sport and physical activity: A review and a look at the future. En G. Tenenbaum \& R. C. Eklund (Eds.), Handbook of sport psychology ( $3^{\mathrm{a}}$ ed., pp. 59-83). New York: Wiley.

Villamarín, F., Maurí, C. \& Sanz, A. (1998). Competencia percibida y motivación durante la iniciación en la práctica del tenis. Revista de Psicología del Deporte, 7(2), 41-56.

Wall, M. \& Côté, J. (2007). Developmental activities that lead to dropout and investment in sport. Physical Education and Sport Pedagogy, 12(1), 77-87. doi: 10.1080/17408980601060358 